PROCEEDINGS OF THE

AMERICAN MATHEMATICAL SOCIETY

Volume 127, Number 6, Pages 1815-1818

S 0002-9939(99)04664-X

Article electronically published on February 17, 1999

\title{
ON THE EXCESS OF SETS OF COMPLEX EXPONENTIALS
}

\author{
NOBUHIKO FUJII, AKIHIRO NAKAMURA, AND RAY REDHEFFER
}

(Communicated by J. Marshall Ash)

\begin{abstract}
For $-\infty<n<\infty$ let $\mu_{n}$ be complex numbers such that $\mu_{n}-n$ is bounded. For $n>0$ define $\lambda_{n}=\mu_{n}+a, \lambda_{-n}=\mu_{-n}-b$ where $a, b \geq 0$. Then the excesses $E$ in the sense of Paley and Wiener satisfy $E\left(\left\{\lambda_{n}\right\}\right) \leq E\left(\left\{\mu_{n}\right\}\right)$.
\end{abstract}

The original sources underlying the problem treated here are [1], [2], [4], and all the results used can be found in the excellent expository account [5], supplemented in a few cases by [3]. In view of this, we do not interrupt the flow by constant reference to the literature.

We use $a, b, c$ for constants and we denote by $\mu=\left\{\mu_{n}\right\}$ a complex sequence defined for $-\infty<n<\infty$. The $L^{2}$ excess of the set $\mu$ in the sense of Paley and Wiener is denoted by $E(\mu)$. If $\left|\mu_{n}-n\right| \leq c$, it is well known that the completeness interval is $[-\pi, \pi]$ and that $E(\mu)$ on this interval is finite. Our object is to prove:

Theorem 1. Let $\left|\mu_{n}-n\right| \leq c$ for $-\infty<n<\infty$ and $\lambda_{0}=\mu_{0}$,

$$
\lambda_{n}=\mu_{n}+a, \quad \lambda_{-n}=\mu_{-n}-b, \quad n>0,
$$

where $a \geq 0$ and $b \geq 0$. Then $E(\lambda) \leq E(\mu)$ on the interval $[-\pi, \pi]$.

For example, suppose $E(\mu)=0$, so $\mu$ is exact on $[-\pi, \pi]$; that is, $\left\{\exp \left(i \mu_{n} x\right)\right\}$ is complete as it stands but becomes incomplete if any term is removed. Then Theorem 1 shows that $\left\{\exp \left(i \lambda_{n} x\right)\right\}$ is either exact or incomplete. Hence it is free in the sense of Schwartz; that is, no exponential $\exp \left(i \lambda_{n} x\right)$ is in the subspace spanned by the others. This always holds if $c<1 / 4$, since the set $\left.\exp \left(i \mu_{n} x\right)\right\}$ forms a basis in that case.

We precede the proof by the following simplifications:

(i) It is easily checked that the completeness properties are unchanged when $\lambda_{n}$ is replaced by $\lambda_{n}-d$, where $d$ is any real constant. Hence there is no loss of generality in taking $b=0$ and replacing $a$ by $a_{0}=a+b$. We write $a_{0}$ instead of $a+b$ because we want $a$ to be a variable, $0 \leq a \leq a_{0}$. This interval describes the relevant values of $a$.

(ii) Since any finite number of terms can be changed without altering the completeness, we assume without loss of generality that

$$
\text { Re } \mu_{n}<0 \text { for } n<0, \quad \operatorname{Re} \mu_{n} \geq 1 \text { for } n>0 .
$$

In particular, $\lambda_{n} \neq 0$ for $n \neq 0$ and all relevant values of $a$. This step and (iii) below may involve increasing $c$, but that does no harm.

Received by the editors January 31, 1997 and, in revised form, September 20, 1997. 1991 Mathematics Subject Classification. Primary 30B60.

(C)1999 American Mathematical Society 
(iii) By adding or removing a finite number of terms $\mu_{n}$ and the $\lambda_{n}$ corresponding thereto, we can ensure that the set $\mu$ is exact.

\section{Proof}

Let

$$
F(a, x)=\prod_{n=1}^{\infty}\left(1-\frac{x}{\mu_{-n}}\right)\left(1-\frac{x}{\mu_{n}+a}\right),
$$

where the factors have been grouped in such a way that the product converges. Since $\mu$ is exact we have $F(0, x) \in L^{2}$, but this would not hold if we had retained the factor corresponding to $\mu_{0}$. Theorem 1 will follow as soon as we show $F(a, x) \in L^{2}$.

Let us therefore consider the integral

$$
I(a)=\int_{-R}^{R+a}|F(a, x)|^{2} d x
$$

where $R$ is a large constant that will later tend to $\infty$. The integral is decomposed in the form

$$
I(a)=\int_{-R}^{-m}+\int_{-m}^{m+a}+\int_{m+a}^{R+a}
$$

where $m \geq 2 c+c^{2}$ is an integer. With $\mu_{-n}=-p-i q$ and $\mu_{n}=r+i s$ for $n \geq m$, the constraint is

$$
(p-n)^{2}+q^{2} \leq c^{2}, \quad(r-n)^{2}+s^{2} \leq c^{2} .
$$

The magnitude squared of the general term in the product is

$$
K(a)=\left|1-\frac{x}{\mu_{-n}}\right|^{2} \frac{(r+a-x)^{2}+s^{2}}{(r+a)^{2}+s^{2}} .
$$

In the first integral (3) we have $x \leq-m$. Differentiation shows that $K^{\prime}(a)$ has the same sign as $s^{2}-(r+a)^{2}+x(r+a)$, which is at most $s^{2}-m$ and hence $\leq 0$. Therefore the first integral is dominated by the corresponding integral with $\mu$, which converges when $R \rightarrow \infty$.

In the second integral $-m \leq x \leq m+a$. Since the integrand is a continuous function of $(a, x)$ the integral is bounded by a constant $C_{m}$ for all relevant values of $a$.

After the substitution $x=y+a$ the third integral is

$$
J(a)=\int_{m}^{R} \prod_{n=1}^{\infty}\left|1-\frac{y+a}{\mu_{-n}}\right|^{2}\left|1-\frac{y+a}{\mu_{n}+a}\right|^{2} d y .
$$

The monotonicity of the integrand

$$
\left|\frac{\mu_{n}-y}{\mu_{-n}}\right|^{2}\left|\frac{\mu_{-n}-a-y}{\mu_{n}+a}\right|^{2}
$$

as a function of $a$ is determined by the second factor,

$$
L(a)=\left|\frac{p+i q+a+y}{r+i s+a}\right|^{2}=\frac{(p+a+y)^{2}+q^{2}}{(r+a)^{2}+s^{2}} .
$$

Evidently $L^{\prime}(a)$ has the same sign as

$$
\left[(r+a)^{2}+s^{2}\right](p+a+y)-\left[(p+a+y)^{2}+q^{2}\right](r+a) .
$$


Now comes an important point. Other variables being fixed, $L^{\prime}(a)$ is largest when $q=0$. Since $q=0$ allows the greatest freedom in the choice of $p$, we see that $q=0$ is in fact the worst case. Setting $q=0$, we divide out the factor $p+a+y$ and find that $L^{\prime}(a) \leq 0$ holds if the function

$$
P=(r+a)^{2}+s^{2}-(p+a+y)(r+a)=(r+a)(r-p-y)+s^{2}
$$

satisfies $P \leq 0$. The two points $(p, q)$ and $(r, s)$ are both in a circle of radius $c$ centered at $(n, 0)$. Hence their distance is at most $2 c$, so $|r-p| \leq 2 c$. Since also $|s| \leq c, r \geq 1$ and $y \geq m \geq 2 c+c^{2}$, it follows that $P \leq 0$. Hence $L^{\prime}(a) \leq 0$ and the integral is maximized when $a=0$. In that case it reduces to the corresponding integral with $\mu$, which converges as $R \rightarrow \infty$. This completes the proof.

\section{Supplementary REMARKS}

The foregoing methods apply to the weighted integral

$$
\int_{-\infty}^{\infty}|F(a, x)|^{2} w(x) d x
$$

where $w(x)$ is any positive continuous function satisfying

$$
0<\liminf _{|x| \rightarrow \infty} \frac{w(x+d)}{w(x)} \leq \limsup _{|x| \rightarrow \infty} \frac{w(x+d)}{w(x)}<\infty, \quad-\infty<d<\infty .
$$

Namely, if the integral converges for $a=0$ then it converges for $a \geq 0$. Admissible weights that are often encountered are

$$
w(x)=e^{h x}, \quad w(x)=\left(1+x^{2}\right)^{h},
$$

where $h$ is a positive or negative constant.

As another generalization, instead of $\left|\mu_{n}-n\right| \leq c$ we could assume that $E(\mu)$ is finite and that

$$
n \operatorname{Re} \mu_{n} \geq 0, \quad\left|\operatorname{Im} \mu_{n}\right| \leq c, \quad\left|\mu_{-n}+\mu_{n}\right| \leq c .
$$

The question whether $|E(\mu)|<\infty \Rightarrow E(\lambda) \leq E(\mu)$ was suggested to one of us by Prof. Lennart Carleson. It appears to be considerably deeper than Theorem 1 and is left open here. The case $p \neq 2$ is also left open, though we do have

$$
E(\lambda) \leq E(\mu)+1
$$

for $1 \leq p \leq \infty$. This follows from the result for $p=2$ and from the fact that changing $p$ changes $E(\lambda)$ by at most 1 .

Theorem 1 is especially plausible when when $\mu=\{n\}$ and $b=0$. In that case the set $\lambda$ for $a=1$ is the same as $\mu-\{1\}$, the set for $a=2$ is the same as $\mu-\{1,2\}$ and so on. Further insight into the case $\mu=\{n\}$ is given by [1], Theorem $\mathrm{V}$ and its proof. The latter suggests a conjecture that is formulated next.

An exponent $p$ satisfying $1<p<\infty$ will be called critical if

$$
1<r<p<q<\infty \Rightarrow E(\lambda, q)>E(\lambda, r) .
$$

When $p=1$ or $p=\infty$ we modify (5) somewhat and instead of $L^{\infty}$ we use the class of absolutely continuous functions that vanish at $\pm \pi$. As $a+b$ increases in Theorem 1, we conjecture that the critical value $p$ in the excess $E(\lambda, p)$ increases steadily to $\infty$, passing through all intermediate values on the way. Upon further increase $E(\lambda, p)$ drops by 1 while $p$ changes to 1 , and the process repeats. 


\section{REFERENCES}

[1] Levinson, Norman, Gap and Density Theorems, AMS Colloquium Publication XXVI (1940), Chapters I, III and IV. MR 2:180d

[2] Paley, Raymond E. A. C and Norbert Wiener, Fourier Transforms in the Complex Domain, AMS Colloquium Publication XIX (1934), Chapter VI. CMP 97:13

[3] Redheffer, Raymond M., Completeness of Sets of Complex Exponentials, Advances in Mathematics 24 (1977) 1-62. MR 56:5852

[4] Schwartz, Laurent, Approximation d'une fonction quelconque par des sommes d'exponentielles imaginaires, Ann. Fac. Sci. Toulouse (1943), 111-176. Reprint (Paris 1959) with some additions.

[5] Young, Robert M., An Introduction to Nonharmonic Fourier Series, Academic Press 1980, Chapter 3. MR 81m:42027

Department of Mathematics, Tokai University, 3-20-1 Orido, Shimizu, Shizuoka 4248610, JAPAN

E-mail address: nfujii@scc.u-tokai.ac.jp

Department of Mathematics, University of California, los Angeles, California, 90095- 1555 